\title{
Rough Contraction Through Partial Meets
}

\author{
Mohua Banerjee and Pankaj Singh \\ Department of Mathematics and Statistics, \\ Indian Institute of Technology, Kanpur 208016, India \\ mohua, pankh@iitk.ac.in
}

\begin{abstract}
The paper addresses the problem of constructing rough belief change functions. Contraction and revision postulates using the notion of rough consequence and rough consistency have been proposed in [2. The base logic is a reasoning framework $\mathcal{L}_{\mathcal{R}}$, that has a semantics of rough truth. We demonstrate here that functions satisfying the contraction postulates can be constructed through the method of partial meets [1]. As a result, a construction of rough revision functions is also obtained.
\end{abstract}

\section{Introduction}

Belief revision models the changes in beliefs of a person when some new information is provided or some earlier information has to be retracted. This kind of study helps in building intelligent agents, who have to manage information about the real world in order to achieve their goals. Beliefs may not represent complete or consistent information in all contexts, so one should be able to revise them when new correct or complete information is acquired. Thus a fundamental issue in belief revision is to decide upon the beliefs which should to be retracted in order to avoid contradiction.

The problem of belief change is well studied in the classical background, where any statement is either true or false - there are well-defined functions which model belief change. But clearly, there is need for appropriate methodology to deal with belief change in situations where information cannot be directly classified into a 'reject' or 'accept' category. Our idea is to be able to express through the base logic, vague/rough statements, and incorporate their properties in belief change. For instance, it is observed that a rough belief and its negation may not lead to a contradiction - motivating a relaxation in the definition of 'contradiction'. In this article, we further investigate an instance of paraconsistent belief change that is based on the logic $\mathcal{L}_{\mathcal{R}}$ of rough truth [2]. $\mathcal{L}_{\mathcal{R}}$ is, in fact, an alternative formulation of the well-known paraconsistent logic $J$ due to Jaśkowski. It should be mentioned that one of the first studies in paraconsistent belief change is presented in [7, where a 4-valued base logic is used. Other work in the direction can be found in 6]9. A comprehensive study on belief change in 3 -valued and rough logics has been recently done in 8 .

Postulates that rough belief change functions should satisfy, have been proposed in 2. In this work, we show that rough contraction functions can be constructed through the method of partial meets [1. Functions are defined using 
maximal subsets of belief sets that do not imply a belief $A$, and it is proved that they satisfy the rough contraction postulates with respect to $A$. The next section gives the requisite preliminaries. Section 3 presents the results on partial meet contraction in the rough context. Section 4 concludes the article.

\section{Preliminaries}

\subsection{Partial Meet Contraction in Classical Belief Change}

The base consequence relation $\vdash$ or operator $C n$ is assumed to be supraclassical (including classical consequence), and to satisfy cut, deduction theorem, monotonicity and compactness [4. A set $K$ of well-formed formulae (wffs) is a belief set, if $K=C n(K)$. The three functions modelling belief change are expansion, revision and contraction. Incoming information may produce new beliefs (which may also lead to inconsistencies). Given a belief set $K$ and a belief $A$, the expansion $K+A$ of $K$ with respect to $A$ is $C n(K \cup\{A\})$. Sets of postulates [14] regulate revision and contraction functions to give belief sets $K * A, K-A$, revised and contracted with respect to a belief $A$ respectively. The contraction and revision functions can be interrelated through the Levi and Harper identities.

One of the methods of constructing a contraction function is through partial meets. The central idea is that a reasonable $K-A$ must not contain $A$. One considers maximal subsets $m$ of $K$ not implying $A$. Formally, $m$ is such that

(i) $m \subseteq K$,

(ii) $A \notin C n(m)$, and

(iii) for any $m^{\prime}$ such that $m \subset m^{\prime} \subseteq K, A \in C n\left(m^{\prime}\right)$.

Let $K \perp A$ denote the collection of such sets $m$, and $M(K)=\bigcup_{A \in K} K \perp A$. Note that if $A \notin K$ then we take $K \perp A=\{K\}$, and if $A$ is a theorem then $K \perp A=\emptyset$. A contraction $K-A$ is then the intersection of some 'preferred' elements of $K \perp A$. Let $S$ denote the selection function for choosing these elements, i.e. $S(K \perp A) \subseteq K \perp A$. A contraction function can then be defined as:

$$
K-A=\left\{\begin{array}{l}
\bigcap S(K \perp A) \text { if } \forall A \\
K \text { otherwise. }
\end{array}\right.
$$

It turns out that the most appropriate $S$ is the one picking the top elements in $K \perp A$, based on a reflexive and transitive preference relation $\geq$ over the maximal subsets in $K \perp A$, i.e.

$$
S(K \perp A)=\left\{m \in K \perp A: m \geq m^{\prime}, \text { for all } m^{\prime} \in K \perp A\right\} .
$$

\section{$2.2 \mathcal{L}_{\mathcal{R}}$}

The notion of rough truth was introduced in [5] to reflect 'inductive' truth, that, with gain of knowledge, leads to total, deductive truth.

The language of the system $\mathcal{L}_{\mathcal{R}}$ is that of a normal modal propositional logic. $\mathcal{L}_{\mathcal{R}}$ is, in fact, based on the modal system $S_{5}$. Let us notice that an $S_{5}$ (Kripke) 
model $\mathcal{M} \equiv(X, R, \pi)$ is essentially an approximation space $(X, R)$, where $X \neq \emptyset$, with the function $\pi$ interpreting every wff of $S_{5}$ as a rough set in $(X, R)$. If $L, M$ denote the necessity and possibility connectives respectively, a modal wff $L \alpha(M \alpha)$, representing 'definitely' ('possibly') $\alpha$, is interpreted by $\pi$ as the lower (upper) approximation $\pi(\alpha) \equiv\left\{x \in X: x^{\prime} \in X\right.$, for all $x^{\prime}$ such that $\left.x R x^{\prime}\right\}$ $\left(\overline{\pi(\alpha)} \equiv\left(\pi(\alpha)^{c}\right)^{c}\right)$ of the set $\pi(\alpha)$.

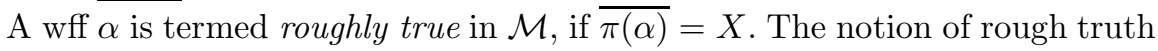
can be extended to rough validity. In the following, $\Gamma$ is any set of wffs.

Definition 1. An $S_{5}$-model $\mathcal{M} \equiv(X, R, \pi)$ is a rough model of $\Gamma$, if and only if every member $\gamma$ of $\Gamma$ is roughly true in $\mathcal{M}$, i.e. $\overline{\pi(\gamma)}=X$.

Definition 2. $\alpha$ is a rough semantic consequence of $\Gamma$ (denoted $\Gamma \approx \alpha$ ) if and only if every rough model of $\Gamma$ is a rough model of $\alpha$. If $\Gamma$ is empty, $\alpha$ is said to be roughly valid, written $\approx \alpha$.

$\mathcal{L}_{\mathcal{R}}$ is defined through the rough consequence relation.

Definition 3. $\alpha$ is a rough consequence of $\Gamma$ (denoted $\Gamma \sim \alpha$ ) if and only if there is a sequence $\alpha_{1}, \ldots, \alpha_{n}(\equiv \alpha)$ such that each $\alpha_{i}(i=1, \ldots, n)$ is either $(i)$ a theorem of $S_{5}$, or (ii) a member of $\Gamma$, or (iii) derived from some of $\alpha_{1}, \ldots, \alpha_{i-1}$ by $R_{1}$ or $R_{2}$ given below. If $\Gamma$ is empty, $\alpha$ is said to be a rough theorem, written $\sim \alpha$.

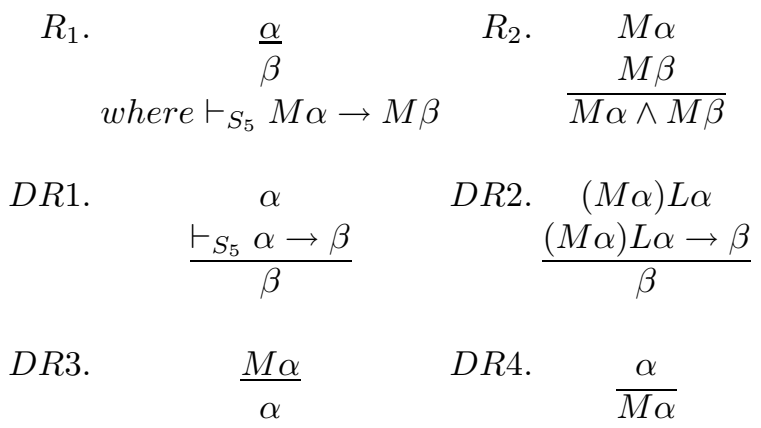

$D R 1-4$ are some derived rules of the system. The usual deduction theorem holds in $\mathcal{L}_{\mathcal{R}}$, though not its converse. $\mathcal{L}_{\mathcal{R}}$ is paraconsistent, i.e. there are $\alpha, \beta$ such that $\{\alpha, \neg \alpha\} \not \mathcal{\beta}$. A set $\Gamma$ is roughly consistent, if $M \Gamma=\{M \alpha: \alpha \in \Gamma\}$ is $S_{5}$-consistent: there is no $\alpha \in \mathcal{L}_{\mathcal{R}}$ such that $M \Gamma \vdash_{S_{5}} \alpha$ and $M \Gamma \vdash_{S_{5}} \neg \alpha$.

\subsection{The Rough Belief Change Postulates}

The changed belief set is denoted as $K *_{r} A\left(K-{ }_{r} A\right)$ representing rough revision (contraction) of $K$ with respect to $A$. The expansion $K+{ }_{r} A$ of $K$ by the wff $A$ is the set $C_{r}(K \cup\{A\})$, where $C_{r}(K)=\{\alpha: K \sim \alpha\}$. The rough equality connective $\approx[3$ is used: $A \approx B \equiv(L A \leftrightarrow L B) \wedge(M A \leftrightarrow M B)$. 


\section{Postulates for Rough Revision}

$K^{*} 1$ For any wff $A$ and belief set $K, K *_{r} A$ is a belief set.

$K^{*} 2 \quad A \in K *_{r} A$.

$K^{*} 3 K *_{r} A \subseteq K+{ }_{r} A$.

$K^{*} 4$ If $\neg A \notin K$, then $K+_{r} A \subseteq K *_{r} A$.

$K^{*} 5$ If $K *_{r} A$ is not roughly consistent, then $\sim \neg A$.

$K^{*} 6$ If $\sim A \approx B$, then $K *_{r} A=K *_{r} B$.

$K^{*} 7 K *_{r}(M A \wedge M B) \subseteq K *_{r} A+_{r} B$.

$K^{*} 8$ If $\neg B \notin K *_{r} A$ then $K *_{r} A+_{r} B \subseteq K *_{r}(M A \wedge M B)$.

\section{Postulates for Rough Contraction}

$K^{-1}$ For any wff $A$ and belief set $K, K{ }_{r} A$ is a belief set.

$K^{-} 2 K-{ }_{r} A \subseteq K$.

$K^{-} 3$ If $A \notin K$, then $K{ }_{-r} A=K$.

$K^{-} 4$ If $A \in K-{ }_{r} A$, then $\sim A$.

$K^{-} 5 K \subseteq\left(K-{ }_{r} A\right)+{ }_{r} A$, if $A$ is of the form $L B$ or $M B$ for some wff $B$.

$K^{-} 6$ If $\sim A \approx B$, then $K-_{r} A=K-_{r} B$.

$K^{-} 7 K{ }_{-r} A \cap K{ }_{r} B \subseteq K-{ }_{r}(M A \wedge M B)$.

$K^{-} 8$ If $A \notin K-{ }_{r}(M A \wedge M B)$ then $K-_{r}(M A \wedge M B) \subseteq K{ }_{-r} A$.

Among other results, we can prove the following (partly done in [2]).

Theorem 1. Let the Levi identity give $*_{r}$, i.e. $K *_{r} A \equiv\left(K-{ }_{r} \neg A\right){ }_{r} A$, where the contraction function $-_{r}$ satisfies $K^{-} 1-8$. Then $*_{r}$ satisfies $K^{*} 1-8$.

Observation 1. Rough belief revision and contraction coincide with the corresponding classical notions if $\vdash_{S_{5}} A \leftrightarrow L A$ for every wff $A$, i.e. all beliefs are definable/describable ( $S_{5}$ collapses into classical propositional logic).

\section{Partial Meet Contraction in Rough Belief Change}

Similar to the classical case, we can construct partial meet contractions in the rough background. We refer to definitions (11), (2) of Section 2.1, modified to use $C_{r}$ or $\sim$. The derived rules $D R 1-4$ (cf. Section 2.2) are used in the proofs.

Lemma 1. Let $m$ be in $K \perp A$ for some $A$ in $K$. Then (i) $m$ is closed, and (ii) $C_{r}(m \cup\{\alpha\})=K$ where $\alpha \in K \backslash m$.

Proof. (i) Let $m \sim \alpha$ and assume $\alpha \notin m$. Now $m \sim \alpha$ implies $m \sim M \alpha$. So $M \alpha \in K$, as $m \subset K$ and $K$ is closed. Hence $m \subset m \cup\{M \alpha\} \subseteq K$, which implies $m \cup\{M \alpha\} \sim A$ or $m \sim M \alpha \rightarrow A$. But then $m \sim A$, as $m \sim M \alpha$ and $M \alpha$ is modal - a contradiction. Hence $\alpha \in m$.

(ii) We already have $C_{r}(m \cup\{\alpha\}) \subseteq K . K \subseteq C_{r}(m \cup\{\alpha\})$ : let $\beta \in K \backslash m$, and we show that $M A \rightarrow M \beta \in m$. Then any belief set containing $M A$ and $m$ (in particular $\left.C_{r}(m \cup\{\alpha\})\right)$ would have $M \beta$ and hence $\beta$. Suppose $M A \rightarrow M \beta \notin m$. Then $m \subset m \cup\{M A \rightarrow M \beta\} \subseteq K$ as $\vdash_{S_{5}} M \beta \rightarrow(M A \rightarrow M \beta)$ and $\beta(M \beta) \in K$. Thus $(M A \rightarrow M \beta) \rightarrow M A \in m$. But $\vdash_{S_{5}}((M A \rightarrow M \beta) \rightarrow M A) \leftrightarrow M A$. So $M A$ or $A \in m$, a contradiction. 
Importantly, Lemma 1(ii) says that all the beliefs in $K$ outside $m$, behave identically with respect to $m$. Further, we have

Lemma 2. Let $A \in K$ and $m \in K \perp A$. Then for all $B$ in $K \backslash m, m \in K \perp B$.

Using this result, we compare $K \perp(M A \wedge M B), K \perp A$ and $K \perp B$. Note that $\{M A \wedge M B\} \sim A, B$, and $\{A, B\} \sim M A \wedge M B$. One can easily see that if $m \in K \perp(M A \wedge M B)$ then it is in $K \perp A$ or $K \perp B$ because $M A \wedge M B \notin m$ implies $A \notin m$ or $B \notin m$. What if $m$ is in $K \perp A$ ? Conditions (i) and (ii) for membership in $K \perp M A \wedge M B$ are satisfied, and Lemma 2 implies (iii). Suppose $m \subset m^{\prime} \subseteq K$. Then in addition to $A \in C_{r}\left(m^{\prime}\right), C_{r}\left(m^{\prime}\right)=K$ also, implying that $B \in C_{r}\left(m^{\prime}\right)($ as $B \in K)$. Hence $M A \wedge M B$ is in $C_{r}\left(m^{\prime}\right)$.

Lemma 3. Let $A$ and $B$ be in $K$. Then $K \perp(M A \wedge M B)=K \perp A \cup K \perp B$.

As $S$ is to select the 'best' elements of $K \perp A$, let us assume that it does so with the help of a reflexive and transitive preference relation $\geq$ over the maximal subsets in $K \perp A$. So $S$ is defined through (2). The following then proves that such a selection works - definition (1) gives a rough contraction function.

Theorem 2. Let $\geq$ be a reflexive and transitive relation on $M(K)$. Then the function defined through (10) with $S$ as in (2) is a rough contraction function, i.e. it satisfies $K^{-1}-8$.

Proof. Let $A \sim \sim B$ abbreviate $\{A\} \sim B,\{B\} \sim A$.

$K^{-1}$ : Each maximal subset $m$ in $S(K \perp A)$ is closed and intersection of closed sets is closed.

$K^{-2}$ : Follows from the definition.

$K^{-} 3$ : If $A \notin K$ then $K \perp A=\{K\}$, and hence $K-{ }_{r} A=K$.

$K^{-} 4$ : If $\not \sim A$ then $K \perp A$ is non-empty. Hence $A \notin K-{ }_{r} A$.

$K^{-} 5$ : In fact, we obtain the classical recovery axiom (viz. $K \subseteq\left(K-{ }_{r} A\right){ }_{r} A$ for any $A$ ), and so, in particular, $K^{-} 5$. Let $\alpha \in K$. Assume $\not A$. We show that $(M A \rightarrow \alpha) \in K-{ }_{r} A$. Using Lemma 11(ii), we get for all $m \in K \perp A$, $C_{r}(m \cup\{A\})=K$, or $C_{r}(m \cup\{M A\})=K$. Thus $M A \rightarrow \alpha \in m$, for all $\alpha \in K$, implying $M A \rightarrow \alpha \in K-{ }_{r} A=\bigcap S(K \perp A)$. Hence $\alpha \in\left(K-{ }_{r} A\right)+A$.

$K^{-}$6: Use, $\sim A \approx B$ implies $K \perp A=K \perp B$. In fact, $A \sim \sim B$ gives the result.

$K^{-}$7: If $\sim A$ or $\sim B$ then obviously this holds: if $\sim A$ then $K-{ }_{r} A=K$ and

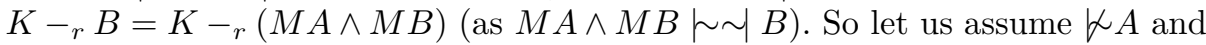
$\not B$. We have to show $(\bigcap S(K \perp A)) \cap(\bigcap S(K \perp B)) \subseteq \bigcap S(K \perp(M A \wedge M B))$. But $S(K \perp A \cup K \perp B)=S(K \perp A) \cap S(K \perp B) \subseteq S(K \perp A) \cup S(K \perp B)$. Hence $\bigcap(S(K \perp A \cup K \perp B)) \supseteq \bigcap(S(K \perp A) \cup S(K \perp B))$. Now $\bigcap(S(K \perp A) \cup S(K \perp B))=$ $(\bigcap S(K \perp A)) \cap(\bigcap S(K \perp B))$. By Lemma 3 we get $(\bigcap S(K \perp A)) \cap(\bigcap S(K \perp B))=$ $\bigcap(S(K \perp A) \cup S(K \perp B)) \subseteq \bigcap(S(K \perp A \cup K \perp B)) \bigcap S(K \perp M A \wedge M B)$.

$K^{-} 8$ : Let $A \notin K-{ }_{r}(M A \wedge M B)$. Thus there exists an $m^{\prime} \in K \perp(M A \wedge M B)$ such that $m^{\prime} \geq m$, for all $m \in K \perp(M A \wedge M B)$ and $A \notin m^{\prime}$. Note that $m^{\prime} \in K \perp A$ as $A \notin m^{\prime}$. Now we show $S(K \perp A) \subseteq S(K \perp(M A \wedge M B))$, whence $\bigcap S(K \perp(M A \wedge$ $M B)) \subseteq \bigcap S(K \perp A)$. If $m^{\prime \prime} \in S(K \perp A)$ then $m^{\prime \prime} \geq m^{\prime} \geq m$, for all $m \in$ $K \perp M A \wedge M B$. By transitivity of $\geq, m^{\prime \prime} \geq m$, for all $m \in K \perp(M A \wedge M B)$. Hence $m^{\prime \prime} \in S(K \perp(M A \wedge M B))$, giving $S(K \perp A) \subseteq S(K \perp(M A \wedge M B))$. 
A rough revision function satisfying all the postulates $K^{*} 1-8$ can thus be obtained through this method as well (using Theorems 2 and 1).

\section{Conclusions}

In rough belief change, the main consideration is to perform the change operations preserving rough consistency and rough truth. It is not surprising that the classical 'exact' notions are achieved as a special case, when a belief $A$ can be identified with $L A$ or $M A$. In this article, we show that the method of partial meets can be used to construct rough contraction and revision functions satisfying all the proposed rough postulates.

The next goal would be to study, in the rough context, the methods of Grove's spheres - focussing on maximally consistent sets of beliefs, and epistemic entrenchment - using an ordering on the beliefs. The latter, in particular, is expected to offer a more computationally tractable solution to the problem of constructing rough belief change functions.

\section{References}

1. Alchourrón, C., Gärdenfors, P., Makinson, D.: On the logic of theory change: partial meet functions for contraction and revision. J. Symb. Logic 50 (1985) 510-530.

2. Banerjee, M.: Rough truth, consequence, consistency and belief revision. In: S. Tsumoto et al., editors, LNAI 3066: Proc. 4th Int. Conf. On Rough Sets and Current Trends in Computing (RSCTC2004), Uppsala, Sweden, June 2004, pages 95-102. Springer-Verlag, 2004.

3. Banerjee, M., Chakraborty, M.K.: Rough consequence and rough algebra. In: W.P. Ziarko, editor, Rough Sets, Fuzzy Sets and Knowledge Discovery, Proc. Int. Workshop on Rough Sets and Knowledge Discovery (RSKD'93), pages 196-207. SpringerVerlag, 1994.

4. Gärdenfors, P., Rott, H.: Belief revision. In: D.M. Gabbay, C.J. Hogger and J.A. Robinson, editors, Handbook of Logic in AI and Logic Programming, Vol.4: Epistemic and Temporal Reasoning, pages 35-132. Clarendon, 1995.

5. Pawlak, Z.: Rough logic. Bull. Polish Acad. Sc. (Tech. Sc.) 35(5-6) (1987) 253-258.

6. Priest, G.: Paraconsistent belief revision. Theoria 67 (2001) 214-228.

7. Restall, G., Slaney, J.: Realistic belief revision. In: Proc. 1st World Congress in the Fundamentals of Artificial Intelligence, Paris, July 1995, 367-378.

8. Singh, P.K.: Belief Revision in Non-classical Logics. Master's Thesis, Indian Institute of Technology, Kanpur, 2005.

9. Tanaka, K.: What does paraconsistency do ? The case of belief revision. In: The Logical Yearbook 1997, T. Childers, editor, Filosophia, Praha, 188-197. 